\title{
Toscana virus encephalitis in Southwest Germany: a retrospective study
}

\author{
R. Dersch ${ }^{1 *}$ (D) A. Sophocleous ${ }^{1}$, D. Cadar², P. Emmerich², J. Schmidt-Chanasit ${ }^{2,3}$ and S. Rauer ${ }^{1}$
}

\begin{abstract}
Background: Toscana virus (TOSV) is an arthropod-borne virus transmitted by phlebotomine sandflies (Phlebotomus sp.) widespread throughout the Mediterranean having the potential to cause meningoencephalitis in humans. In Germany, the vectors of TOSV are introduced recently and become endemic especially in Southwestern Germany. As TOSV is not investigated regularly in patients with meningoencephalitis, cases of TOSV-neuroinvasive disease may remain mostly undetected.

Methods: We conducted a retrospective cohort study on patients with meningoencephalitis without identification of a causal pathogen from 2006 to 2016. Serologic assessment for anti-TOSV-IgG and IgM was performed on serum and CSF. Demographic, clinical and CSF data from TOSV-positive patients were compared to a cohort of patients with meningoencephalitis due to enterovirus. Informed consent was obtained from all included patients.

Results: We found 138 patients with meningoencephalitis without identified causal pathogen. From 98 of these patients CSF and serum was available for further testing. Additionally, we included 27 patients with meningoencephalitis due to enterovirus. We identified two patients with serological confirmed TOSV-neuroinvasive disease (TOSV-IgM and IgG positive, 2\%) and two patients with possible TOSV-neuroinvasive disease (isolated TOSV-IgM positive, 2\%). Overall, TOSV-neuroinvasive was detected in $4 \%$ of our cases with suspected viral meningoencephalitis. None of them had a history of recent travel to an endemic area.
\end{abstract}

Conclusions: We found cases of TOSV-neuroinvasive disease in our German cohort of patients with meningoencephalitis. As no recent history of travel to an endemic area was reported, it remains probable that these cases resemble autochthonous infections, albeit we cannot draw conclusions regarding the origin of the respective vectors. TOSV could be considered in patients with meningoencephalitis in Germany.

Keywords: Emerging viruses, Encephalitis, Meningitis, Sandfly fever, Toscana virus, Neuroinfectious diseases

\section{Background}

Viral meningoencephalitis is suspected in patients with altered mental status, headache, fever and cerebrospinal fluid (CSF) pleocytosis often accompanied by neurological deficits, seizures and alterations in cerebral imaging or electroencephalography (EEG). The most

*Correspondence: Rick.Dersch@uniklinik-freiburg.de

${ }^{1}$ Clinic of Neurology and Neurophysiology, Medical Center - University of Freiburg, Faculty of Medicine, University of Freiburg, 79106 Freiburg, Germany

Full list of author information is available at the end of the article common viral pathogens causing meningoencephalitis in central Europe are considered the herpes simplex virus, varicella-zoster virus, enteroviruses and tick-borne encephalitis virus [1]. There are considerable regional differences, especially regarding arthropod-borne transmission, which relates closely to the habitat of the respective arthropod vector.

However, in patients with suspected viral meningoencephalitis the causative pathogen cannot be identified in approximately 50\% [2]. In the Mediterranean area, Toscana virus (TOSV) is one for the most prevalent viruses in patients with meningoencephalitis [3]. Toscana virus is 
an arthropod-borne enveloped, negative-stranded RNA virus transmitted by sandflies (Phlebotomus sp.) and belongs to the Phlebovirus genus within the Phenuiviridae family. In humans, infections with TOSV are usually asymptomatic or mild, but can also cause neuroinvasive disease called sandfly fever. In Central Italy, TOSV is responsible for approximately $80 \%$ of the cases of aseptic meningitis during the summer [4]. The incubation period is usually 3-7 days, but can be as long as 2 weeks. Typical symptoms are headache, fever, neck rigidity, myalgia, photophobia and focal neurological signs [3]. In most cases, symptoms resolve after 7-10 days of febrile illness. However, severe courses of disease and cases of fatal encephalitis have been reported previously $[5,6]$. In cases of acute TOSV-infection, usually -TOSV-IgM and -IgG are detectable in serum. IgM decreases over time and cease to be detectable whereas IgG can be detected years after contact with TOSV [7].

In Germany, cases of sandfly fever caused by TOSV are rare and usually occur in travelers after exposure in endemic areas [5, 8]. However, sandflies from the Phlebotomus species are increasingly detected in areas where these vector have not been reported previously, especially in Southwest Germany in the Upper Rhine Valley [9]. Sandfly populations can now be found over several years at the same site [9]. Thus, autochthonous populations can be assumed. Climate change with an elevation of the annual mean temperature over the last decades is discussed as a relevant factor for the expansion of the habitat of sand flies [9].

As competent vectors of TOSV are present in southwestern Germany, an elevated risk of transmission of this virus to humans seems possible. As awareness of TOSV is currently low, TOSV is usually not incorporated in the diagnostic work-up of patients with viral meningoencephalitis in Germany. As a causal pathogen cannot be identified in approximately $50 \%$ of cases with suspected viral encephalitis, it is possible that cases of TOSV-neuroinvasive disease remain undetected. To identify possible undetected cases of TOSV-neuroinvasive disease, we performed a retrospective cohort study on patients who were diagnosed as having a suspected viral meningoencephalitis without identified pathogen. We serologically tested CSF and serum for TOSV IgM and IgG. As TOSV-cases are undistinguishable from other viral pathogens regarding clinical features, we investigated whether there are specific CSF profiles or laboratory test results that could guide diagnostic assessment in cases of viral meningoencephalitis in the future.

\section{Methods}

We searched medical records of a tertiary care center in Southwest Germany (Clinic of Neurology and Neurophysiology, Medical Center - University of Freiburg) from January 2006 - December 2016 for patients with suspected viral meningoencephalitis (ICD 10-code A87). For being considered as having possible viral meningoencephalitis, patients had to exhibit headache, altered mental state, fever and CSF pleocytosis. Patients were excluded if a causal pathogen was identified (diagnostic workup includes PCR for herpes simplex virus, varicella zoster virus, Enterovirus, eubacterial PCR, PCR for Listeria as well as serology for B. burgdorferi, syphilis and tick-borne-encephalitis virus) or onconeural antibodies (Hu, Yo, Ri, CV2/CRMP5, Amphiphysin, Ma1, Ma2, SOX1, GAD65, Tr (DNER), Zic4) or antibodies against neuronal cell-surface antigens (NMDAR, LGI1, AMPAR, CASPR2, GABAR) were detected. To investigate clinical spectrum and the profile of changes in CSF, data on clinical course and results from laboratory testing as well as CSF analysis were collected. A control group was compiled of patients with aseptic meningoencephalitis due to infection with enterovirus confirmed with PCR. After initial diagnostic workup, CSF and serum was stored for further investigation at $-80^{\circ} \mathrm{C}$. CSF and serum samples were stored, treated, and analyzed in the same manner for all included patients. Written informed consent was obtained from each patient. The study was conducted according to Helsinki criteria and received approval by the local ethics committee of the Medical Center - University of Freiburg, Faculty of Medicine (EK FR 536/19). Demographical data and information on initial symptoms, treatment and course of disease as well as data on CSF analysis and laboratory analyses were obtained from the medical records.

Serological testing for anti-TOSV-IgG and IgM and PCR for Phlebovirus-RNA was performed at the Bernhard-Nocht-Institute for Tropical Medicine, Hamburg, Germany [10]. Immunofluorescence assays (IFA) for various sandfly-borne phleboviruses (TOSV, SFNV and SFSV) were performed with virus-infected Vero E6 cells. In brief, Vero cells were spread onto slides, air dried, and fixed in acetone. Serum samples were serially diluted in phosphate-buffered saline (PBS) starting with an initial dilution of 1:10, added to the cells, and incubated for $90 \mathrm{~min}$ at $37^{\circ} \mathrm{C}$. After washing with PBS, slides were incubated with Fluoresceine isothiocyanate-labeled rabbit anti-human IgG and IgM antibodies (SIFIN, Berlin, Germany) at $37^{\circ} \mathrm{C}$ for $25 \mathrm{~min}$. IgG titers or IgM titers of $1: 20$ or more were considered positive.

All samples were analyzed in triplets. For a diagnosis of definite/confirmed TOSV-infection evidence of anti-TOSV-IgM and -IgG was required [7]. Cases with isolated anti-TOSV IgM antibodies were regarded as possible TOSV-infections. Cases with isolated anti-TOSV IgG antibodies most likely represent residual antibody 
titers after a TOSV-infection in the past with no relation to the acute presentation of the patient.

\section{Results}

We found 138 patients with suspected viral meningoencephalitis without identified causal pathogen. Of these patients, serum or CSF was available from 98 patients for further testing, 40 patients without available specimen were excluded from further analysis. In the control group of patients with meningoencephalitis due to enterovirus we included 27 patients. In the group of patients with suspected viral meningoencephalitis of unknown origin we could identified 2 cases with definite TOSV-infection
(anti-TOSV-IgM and IgG TOSV positive), 2 cases with possible TOSV-infection (isolated anti-TOSV-IgM positive) and 2 cases with residual antibody titers (isolated anti-TOSV-IgG positive). One patient with definite TOSV-infection also showed positive anti-TOSV-IgG in CSF. In the two patients with both anti-TOSV-IgM and anti-TOSV-IgG antibodies follow up testing 1-2 weeks after baseline showed increasing titers for both IgM and IgG. Unfortunately, in patients with only anti-TOSV-IgM antibodies no material was available for follow-up serology. PCR for RNA of Phleboviruses was negative in all samples. Demographic and clinical data for the included patients are shown in Table 1. None of the TOSV-patients

Table 1 Demographical and clinical characteristics and results from CSF analysis of included patients. For groups, values resemble mean with SD in brackets

\begin{tabular}{|c|c|c|c|c|c|c|}
\hline & Case 1 & Case 2 & Case 3 & Case 4 & $\begin{array}{l}\text { TOSV overall } \\
(n=4)\end{array}$ & Enterovirus $(n=27)$ \\
\hline $\begin{array}{l}\text { TOSV-Antibiodies } \\
\text { in serum (base- } \\
\text { line) }\end{array}$ & $\begin{array}{l}\text { IgM: } 1 / 320 \\
\text { lgG: } 1 / 1280\end{array}$ & $\begin{array}{l}\lg M: 1 / 1280 \\
\lg G: 1 / 320\end{array}$ & $\lg M: 1 / 160$ & lgM: 1/80 & & \\
\hline $\begin{array}{l}\text { TOSV-Antibio- } \\
\text { dies in serum } \\
\text { (1-2 weeks) }\end{array}$ & $\begin{array}{l}\operatorname{lgM}: 1 / 1280 \\
\operatorname{lgG:} 1 / 5120\end{array}$ & $\begin{array}{l}\text { IgM: } 1 / 640 \\
\text { lgG: } 1 / 640\end{array}$ & n.a. & n.a. & & \\
\hline $\begin{array}{l}\text { TOSV-Antibodies } \\
\text { in CSF }\end{array}$ & $\operatorname{lgG}(1: 20)$ & n.a. & negative & negative & & \\
\hline TOSV diagnosis & definite & definite & possible & possible & & \\
\hline Age [yrs] & 70 & 21 & 71 & 80 & $60.5(26.7)$ & 35 \\
\hline Sex & $\mathrm{m}$ & $f$ & $f$ & $f$ & & $14 \mathrm{f}: 13 \mathrm{~m}$ \\
\hline $\begin{array}{l}\text { Clinical presenta- } \\
\text { tion }\end{array}$ & $\begin{array}{l}\text { meningoencepha- } \\
\text { litis }\end{array}$ & meningitis, myelitis & $\begin{array}{l}\text { meningoencepha- } \\
\text { litis }\end{array}$ & $\begin{array}{l}\text { meningoencepha- } \\
\text { litis }\end{array}$ & & meningitis (100\%) \\
\hline CSF cell count $[/ \mu \mathrm{l}]$ & 162 & 681 & 10 & 53 & $226.5(309.7)$ & $319.8(395.6)$ \\
\hline $\begin{array}{l}\text { Total protein [mg/ } \\
\text { dl] }\end{array}$ & 736 & 3460 & 612 & 498 & $1326.5(1425.7)$ & $60.7(21.9)$ \\
\hline$A Q^{a}$ & 9.4 & 52.3 & 2.67 & 7.9 & $18.1(23.0)$ & $7.2(2.8)$ \\
\hline $\begin{array}{l}\text { Intrathecal } \\
\text { Immunoglobulin- } \\
\text { synthesis }\end{array}$ & $\lg M$ & none & none & none & & $\begin{array}{l}\lg M n=2 \\
\lg A n=2\end{array}$ \\
\hline Lactate $[\mathrm{mmol} / \mathrm{l}]$ & 3.62 & 4.93 & 2.67 & 1.91 & $3.3(1.3)$ & $2.2(0.6)$ \\
\hline CSF cytology & $\begin{array}{l}\text { lymphocytes 90\%, } \\
\text { monocytes 10\% } \\
\text { activated lympho- } \\
\text { cytes + }\end{array}$ & $\begin{array}{l}\text { granulocytes } 60 \% \\
\text { lymphocytes } 30 \% \\
\text { monocytes } 10 \%\end{array}$ & $\begin{array}{l}\text { lymphocytes } 90 \% \\
\text { monocytes } 10 \%\end{array}$ & $\begin{array}{l}\text { lymphocytes: } 90 \% \\
\text { monocytes: } 10 \%\end{array}$ & & \\
\hline $\begin{array}{l}\text { MRI/CT abnor- } \\
\text { malities }\end{array}$ & none & none & none & none & none & none \\
\hline$W_{B C}{ }^{a}$ & 5.52 & 6.6 & 9.0 & 7.8 & $7.23(1.5)$ & $8.1(2.5)$ \\
\hline $\mathrm{CRP}\left[\mathrm{mg} / \mathrm{l}^{\mathrm{a}}\right.$ & $<3$ & 8 & 9 & 53 & $23.3(25.7)$ & $13.7(14.1)$ \\
\hline Transaminases $^{a}$ & normal & $\begin{array}{l}\text { AST, ALT, GGT } \\
\text { elevated }\end{array}$ & normal & normal & & $\begin{array}{l}\text { 1/27 elevated GGT/ } \\
\text { ALT }\end{array}$ \\
\hline $\begin{array}{l}\text { Lenght of stay in } \\
\text { hospital [days] }\end{array}$ & 7 & 18 & 14 & 16 & $13.75(4.8)$ & $5.5(2.8)$ \\
\hline Clinical outcome & no sequelae & $\begin{array}{l}\text { Residual parapare- } \\
\text { sis, slight cognitive } \\
\text { impairment }\end{array}$ & no sequelae & no sequelae & $1 / 4$ (25\%) sequelae & $\begin{array}{l}3 / 27(11 \%) \text { sequelae } \\
\text { (headache } n=1 \\
\text { fatigue } n=2)\end{array}$ \\
\hline
\end{tabular}

${ }^{a} A Q$ albumin quotient, $W B C$ white blood cell count, CRP C-reactive protein, $A S T$ aspartat transaminase, $A L T$ alanin transaminase, GGT gamma-glutamyltransferase 
had a history of travel to regions with known endemic TOSV-infection.

One patient (case 2) had a severe affection of the CNS with myelitis and prolonged recovery requiring inpatient rehabilitation treatment. No specific imaging abnormalities were found in patients with TOSV-neuroinvasive disease.

Due to the small sample size of patients with TOSVneuroinvasive disease, statistical comparisons with patients with meningoencephalitis due to enterovirus were not deemed justified.

\section{Discussion}

In our cohort of 98 patients with suspected viral meningoencephalitis with unknown origin we found 2 cases of definite TOSV-neuroinvasive disease (2\%) and 2 cases of possible TOSV-neuroinvasive disease (2\%). Overall, TOSV antibodies were detected in $4 \%$ of our cases with suspected viral meningoencephalitis with unknown origin. One patient had severe affection of the CNS with myelitis, all other patients had a mild course of disease. The individual patients did not reported recent travel to regions with endemic TOSV-infection. Therefore, these cases are likely to represent autochthonous cases of TOSV-infection. In our retrospective study, we are unable to distinguish whether the infection was acquired from a local population of vectors or from migrating vectors (e.g. introduced in cars from travelers from endemic regions).

Due to the small sample size of patients with TOSVneuroinvasive disease, statistical comparison with patients with meningoencephalitis due to enterovirus were not justified. However, regarding data from CSF analysis and laboratory analysis, no distinct pattern regarding TOSV-neuroinvasive disease emerges.

As our study was retrospective and had a descriptive design, several limitations have to be discussed. Patients were screened based on the diagnosis of suspected viral meningoencephalitis in the initial medical records. We may have missed eligible patients that were classified otherwise e.g. as fever of unknown origin or post-infectious headache.

Surplus CSF and serum samples were stored at $-80^{\circ} \mathrm{C}$, with potentially disadvantageous effects on sample quality. A prospective study with timely initiation of diagnostic procedures would be desirable to enhance quality of samples and enable further diagnostic procedures like PCR. Two of the three patients with possible TOSV-neuroinvasive disease had only evidence of anti-TOSV IgM. A follow-up investigation regarding seroconversion would have been informative but was unfortunately not possible as patients were unavailable for further evaluation. Whether these cases resemble true TOSV-infections or were due to crossreacting antibodies remains unclear. Recently, we demonstrated that a bacterial-induced immunopathological state mimicked acute sandfly fever and led to unspecific low TOSV-IgM and -IgG titers in IFA [11]. However, low unspecific TOSV IFA titers were excluded in our study.

PCR for RNA of Phlebovirus was negative in all samples. However, as viremic period is short in individuals infected with TOSV, negative PCR is of limited use in diagnosing TOSV-neuroinvasive disease.

Other case series on patients with TOSV neuroinvasive disease report similar CSF findings and also a small proportion of patients with severe CNS involvement [12]. Some cases of hydrocephalus in the course if TOSV neurinvasive disease have been reported, however none of our patients had signs of hydrocephalus in cerebral imaging [13, 14]. Previous studies on CSF in TOSVneuroinvasive disease showed moderate lymphocytic CSF pleocytosis and moderate elevation of CSF protein [12]. CSF characteristics in our patients with TOSVneuroinvasive disease were similar to those previous reports, albeit these findings remain indistinguishable from other causes of viral meningoencephalitis. Case 2 showed the highest CSF pleocytosis and a mixed cell pleocytosis with high fraction of granulocytes. This may resemble the larger extend of neuroinflammation in this case with severe spinal involvement. Anti-TOSV-IgG in CSF were only detectable in one patient and negative in two patients. This may indicate that TOSV induces an acute systemic inflammation similar to immune responses seen in other neurozoonotic diseases, like tick-borne-encephalitis.

Our study shows that autochthonous cases of meningoencephalitis due to TOSV infection may exist in Germany. Information on distribution of viral pathogens may influence clinical decisions on diagnostic approaches when faced with patients with suspected viral meningoencephalitis. Dissemination of viral pathogens may also be relevant regarding measures of sandfly control, as such measures are already in place for other arthropod vectors of emerging viruses like Aedes spec [15].. Identification of patients with TOSV-neuroinvasive disease could have implications for public health, as specific vector control programs could be considered around a diagnosed patient.

Despite usually favorable courses of disease, severe cases with parenchymal involvement and residual neurological symptoms may occur.

Further prospective studies could generate more information on prevalence of TOSV-neuroinvasive disease in Germany, especially in areas at-risk at developing established populations of sand flies. 
As currently no treatment or vaccine is available, no specific treatment can be offered to patients diagnosed with TOSV-neuroinvasive disease. However, identifying TOSV as the causal agent in a patient with meningoencephalitis can help to avoid unnecessary treatment with antibiotics and antiviral agents [16].

As prevalence is expected to rise, TOSV should be considered as a possible pathogen by clinicians in Germany when faced with patients with viral meningoencephalitis [9].

\section{Acknowledgements}

Not applicable.

\section{Authors' contributions}

RD: conception of the study, acquisition and interpretation of data. AS: recruiting participants and acquisition of data. DC: analysis of samples, interpretation of data. PE: analysis of samples, interpretation of data. JSC: conception of the study, supervision of analysis of samples, interpretation of data. SR: conception of the study, interpretation of data. All authors have approved the submitted manuscriped and have agreed both to be personally accountable for the author's own contributions.

\section{Funding}

Open Access funding enabled and organized by Projekt DEAL.

\section{Availability of data and materials}

The data that support the findings of this study are available on reasonable request from the corresponding author.

\section{Declarations}

\section{Ethics approval and consent to participate}

The study was conducted according to Helsinki criteria and received approval by the local ethics committee of the Medical Center - University of Freiburg, Faculty of Medicine (EK FR 536/19). Written informed consent was obtained from each patient.

\section{Consent for publication}

Not applicable.

\section{Competing interests}

$\mathrm{RD}$ reports lecture fees from Roche and Alexion and travel grants by Biogen. AS declares no conflict of interest.

DC declares no conflict of interest.

PE declares no conflict of interest.

JSC declares no conflict of interest.

SR reports consulting and lecture fees, grant and research support from Bayer Vita GmbH, Biogen, Celgene, Merck Serono, Novartis, Sanofi- Aventis, Genzyme, Roche and Teva; founding executive board member of ravo Diagnostika $\mathrm{GmbH}$ Freiburg.

\section{Author details}

${ }^{1}$ Clinic of Neurology and Neurophysiology, Medical Center - University of Freiburg, Faculty of Medicine, University of Freiburg, 79106 Freiburg, Germany. ${ }^{2}$ WHO Collaborating Centre for Arbovirus and Haemorrhagic Fever Reference and Research, Bernhard Nocht Institute for Tropical Medicine, 20359 Hamburg, Germany. ${ }^{3}$ Faculty of Mathematics, Informatics and Natural Sciences, Universität Hamburg, 20148 Hamburg, Germany.

Received: 26 October 2021 Accepted: 14 December 2021 Published online: 22 December 2021

\section{References}

1. Boucher A, Herrmann JL, Morand P, Buzelé R, Crabol Y, Stahl JP, et al. Epidemiology of infectious encephalitis causes in 2016. Med Mal Infect. 2017:47(3):221-35.
2. Tyler KL. Acute Viral Encephalitis. N Engl J Med. 2018;379(6):557-66.

3. Charrel RN, Bichaud $L$, de Lamballerie X. Emergence of Toscana virus in the mediterranean area. World JVirol. 2012;1(5):135-41.

4. Valassina $\mathrm{M}$, Cusi MG, Valensin PE. A Mediterranean arbovirus: the Toscana virus. J Neuro-Oncol. 2003;9(6):577-83.

5. Bartels S, de Boni L, Kretzschmar HA, Heckmann JG. Lethal encephalitis caused by the Toscana virus in an elderly patient. J Neurol. 2012 Jan;259(1):175-7.

6. Baldelli F, Ciufolini MG, Francisci D, Marchi A, Venturi G, Fiorentini C, et al. Unusual presentation of life-threatening Toscana virus meningoencephalitis. Clin Infect Dis Off Publ Infect Dis Soc Am. 2004;38(4):515-20.

7. Pierro A, Ficarelli S, Ayhan N, Morini S, Raumer L, Bartoletti M, et al. Characterization of antibody response in neuroinvasive infection caused by Toscana virus. Clin Microbiol Infect Off Publ Eur Soc Clin Microbiol Infect Dis. 2017;23(11):868-73.

8. Imirzalioglu C, Schaller M, Bretzel RG. Sandfly fever Naples virus (serotype Toscana) infection with meningeal involvement after a vacation in Italy. Dtsch Med Wochenschr. 2006;131(50):2838-40.

9. Oerther S, Jöst H, Heitmann A, Lühken R, Krüger A, Steinhausen I, et al. Phlebotomine sand flies in Southwest Germany: an update with records in new locations. Parasit Vectors. 2020;13(1):173.

10. Weidmann M, Sanchez-Seco MP, Sall AA, Ly PO, Thiongane Y, Lô MM, et al. Rapid detection of important human pathogenic Phleboviruses. J Clin Virol. 2008:41(2):138-42.

11. Tappe D, Schmidt-Chanasit J, Günther S, Ries A, Ziegler U, Müller A, et al. Acute Toscana virus infection mimicked by Yersinia-induced reactive arthritis syndrome after journey to Spain. J Clin Virol. 2010;47(1):104-5.

12. Jaijakul S, Arias CA, Hossain M, Arduino RC, Wootton SH, Hasbun R. Toscana meningoencephalitis: a comparison to other viral central nervous system infections. J Clin Virol Off Publ Pan Am Soc Clin Virol. 2012;55(3):204-8.

13. Oechtering J, Petzold GC. Acute hydrocephalus due to impaired CSF resorption in Toscana virus meningoencephalitis. Neurology. 2012;79(8):829-31.

14. Charrel RN, Petzold GC, Oechtering J. Acute hydrocephalus due to impaired CSF resorption in Toscana virus meningoencephalitis. Neurology. 2013;80(15):1444.

15. Werner D, Kowalczyk S, Kampen $\mathrm{H}$. Nine years of mosquito monitoring in Germany, 2011-2019, with an updated inventory of German culicid species. Parasitol Res. 2020;119(9):2765-74.

16. Veater J, Mehedi F, Cheung CK, Nabarro L, Osborne J, Wong N, et al. Toscana virus meningo-encephalitis: an important differential diagnosis for elderly travellers returning from Mediterranean countries. BMC Geriatr. 2017;17(1):193.

\section{Publisher's Note}

Springer Nature remains neutral with regard to jurisdictional claims in published maps and institutional affiliations. 